\section{Severe immune thrombocy- topenia induced by a single dose of nivolumab in a patient with advanced non-small cell lung cancer}

\author{
Adam Khorasanchi, Roger Keresztes \\ Department of Medicine, Stony Brook \\ University Hospital, NY, USA
}

\begin{abstract}
Nivolumab-induced immune thrombocytopenia (ITP) is a rare process with few reported cases. We present a 67-year-old man with advanced non-small cell lung cancer who was hospitalized with severe thrombocytopenia. Physical exam was notable for petechiae across his chest and extremities as well as bullae in his oral cavity. The patient initially received high-dose glucocorticoids and intravenous immunoglobulin, but did not respond to treatment. He was then started on weekly rituximab and after three doses, there was complete resolution of his thrombocytopenia. Altogether, his presentation was an extreme case and rare side effect of immune checkpoint therapy, known as nivolumab-induced ITP. Diagnosis of nivolumab-induced ITP is challenging given the lack of specific testing and a wide differential diagnosis. There are few cases reporting severe ITP following nivolumab treatment. We highlight the importance of recognizing and treating this rare complication of immunotherapy.
\end{abstract}

\section{Introduction}

Lung cancer is the leading cause of cancer death worldwide. Nivolumab, an antiPD1 immune checkpoint inhibitor (ICI) has been approved for use in a wide variety of malignancies including advanced non-small cell lung cancer (NSCLC). ICIs can provide substantial therapeutic benefit, however many immune-related adverse events (irAEs) have also emerged. Mechanistically, ir-AEs are thought to be caused by a reinvigoration of exhausted T-cells, which evoke inflammation and ultimately lead to their toxicity. Other immune cells may also play a role, including $B$ cells that produce antibodies and mediate the toxicity. ${ }^{1,2}$

While virtually any organ system can be affected by ICIs, the dermatologic, gastrointestinal tract, and endocrine systems are most commonly involved. ${ }^{1}$ Involvement of the hematopoietic system is rare, but important to recognize as it can be associated with life-threatening outcomes. In a 2019 study, grade 2 or worse hematologic ir-AEs were noted in thirty-five patients or $3.7 \%$ of the study population. ${ }^{3,4}$ In this paper, we present a rare case of severe ITP induced by a single dose of nivolumab in a patient with advanced NSCLC and review the current literature.

\section{Case Report}

A 67-year-old man who was a former tobacco smoker (100 pack-year history) presented to our oncology clinic following a diagnosis of Stage IIIa NSCLC. He had a past medical history significant for ulcerative colitis, which was well-controlled on balsalazide with no recent flares. He was initially noted to have a lung nodule on a surveillance computed tomography (CT) scan for abdominal aortic aneurysm. A positron emission tomography (PET) scan was done, which demonstrated a metabolically active right lower lobe lesion measuring $9.1 \times 3.9$ centimeter with an extension to the hilum. Enlarged right paratracheal lymph nodes were also noted be metabolically active. He underwent endobronchial ultrasound guided fine needle aspiration of the mass and mediastinal lymph nodes. Results were consistent with squamous cell carcinoma with metastases in the lymph nodes. The pathological diagnosis was T3N2M0 stage IIIa squamous cell lung carcinoma. PET scan at the time did not demonstrate any evidence of extra thoracic metastatic disease and therefore he was advised to undergo neoadjuvant chemotherapy followed by restaging of mediastinum for consideration of curative surgical resection. The patient was deemed to be of good functional status and started on paclitaxel, carboplatin. After a single course of chemotherapy, he was hospitalized with near fatal sepsis. He was treated with intravenous (IV) vancomycin and Zosyn, followed by levofloxacin to complete a fourteen-day course. Following his recovery, his case was discussed at our institutional tumor board and given the severe toxicity and low likelihood chemotherapy would make him operable, the patient was started on a course of concurrent chemoradiation. He tolerated this treatment well until the end of his six-week course when he developed febrile neutropenia and was found to have $\mathrm{C}$. difficile colitis. He was treated with IV cefepime, followed by Augmentin to complete a ten-day course as well as fourteen days of oral vancomycin. CT scan during this hospitalization showed a new liver lesion, which was proven by biopsy to be
Correspondence: Adam Khorasanchi, Department of Internal Medicine, Health Science Center T16, Room 020, Stony Brook, NY 11790-8160, USA.

Tel.: +1.(631).444.7411 - Fax: +1.(631).444.2493.

E-mail: adam.khorasanchi@stonybrookmedicine.edu

Key words: Nivolumab; immune checkpoint inhibitor; non-small cell lung cancer; immunerelated thrombocytopenia.

Contributions: the authors contributed equally

Conflict of interest: the authors declare no potential conflict of interest.

Ethics approval: No approval is required.

Informed consent: Informed consent was unable to be obtained, however no identifiable information about the patient was included in the publication of this manuscript

Received for publication: 26 February 2020.

Revision received: 14 June 2020.

Accepted for publication: 15 June 2020.

This work is licensed under a Creative Commons Attribution NonCommercial 4.0 License (CC BY-NC 4.0).

CCopyright: the Author(s), 2020

Licensee PAGEPress, Italy

Clinics and Practice 2020; 10:1249

doi:10.4081/cp.2020.1249

metastatic squamous cell carcinoma.

$\mathrm{He}$ was subsequently started on immunotherapy nivolumab $3 \mathrm{mg} / \mathrm{kg}$ every two weeks. Two weeks following his first nivolumab administration, the patient was noted to have a platelet count of $1000 / \mu \mathrm{L}$ (previously $188,000 / \mu \mathrm{L}$ ) as well as petechiae on his arms and legs. His platelet count was repeated in a citrate tube and confirmed on peripheral smear. His hemoglobin and white blood cell count remained unchanged compared to prior laboratory results. Based on his severe thrombocytopenia, he was admitted to the hospital urgently that same day. On arrival, he was hemodynamically stable. Physical exam was notable for petechiae across his chest and extremities as well as bullae in his oral cavity. Given the acute drop in his platelet count and recent immunotherapy exposure, he was diagnosed with a rare ir-AE, known as nivolumab-induced ITP. Platelet-associated immunoglobulin G antibody levels (PA$\mathrm{IgG})$ were not measured. He was initially started on IV steroids and received three doses of IV immunoglobulin (IVIG). His platelet count had increased to $37,000 / \mu \mathrm{L}$. At this point, the patient was switched to 
oral prednisone. However, his platelet count decreased to $18,000 / \mu \mathrm{L}$. He was then started on weekly rituximab and after three doses, his platelet count recovered to $150,000 / \mu \mathrm{L}$.

Following platelet count recovery, the patient was enrolled in our clinical trial and started on an antibody-drug conjugate as treatment for his advanced NSCLC. One week after receiving his first dose, he was readmitted to the hospital for neutropenic fever and respiratory distress. He was treated for pneumonia as well as suspected radiation pneumonitis. Despite treatment, his respiratory status worsened and after a goals of care discussion with his family, the decision was made to pursue comfort measures. Unfortunately, the patient died fourteen days following hospital admission.

\section{Discussion}

Nivolumab is a fully human immunoglobulin G4 (IgG4) monoclonal antibody that selectively inhibits programmed cell death-1 (PD-1) activity by binding to the PD-1 receptor and blocking its interaction with both PD-L1 and PD-L2. PD-1 receptor blockade removes inhibitory signals of T-cell activation, thus enabling tumor-reactive $\mathrm{T}$ cells to overcome regulatory mechanisms and mount an effective anti-tumor response. This anti-tumor response is manifested through reinvigora-

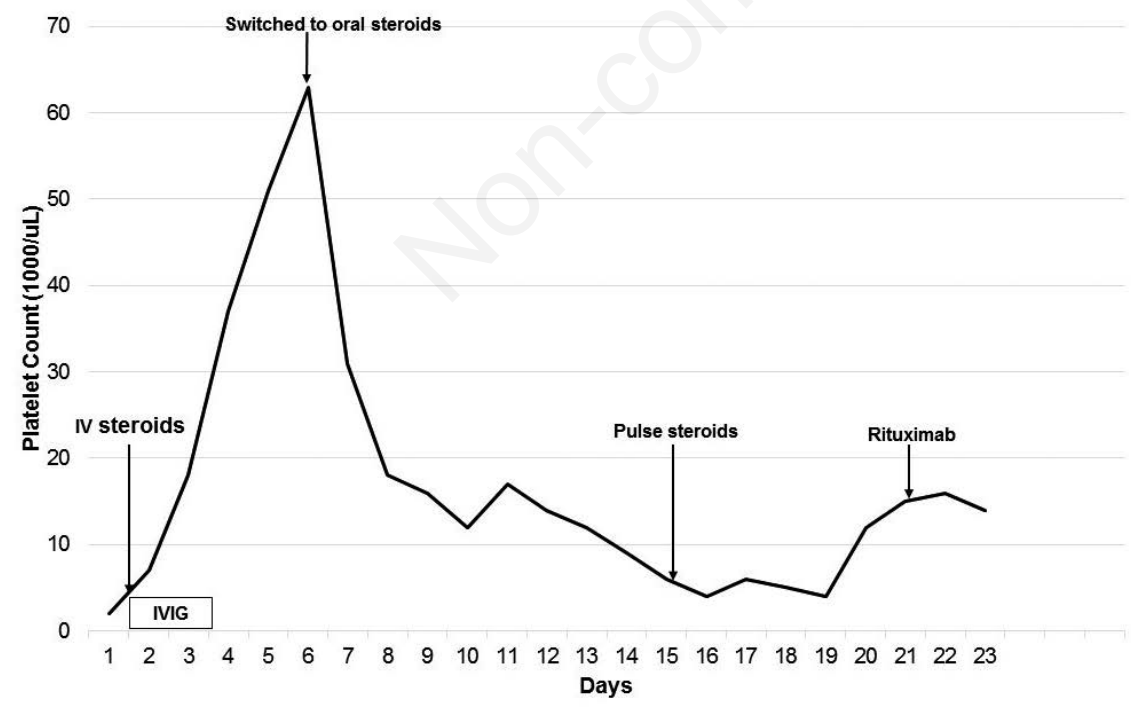

Figure 1. Clinical course of the present case. The patient was hospitalized with severe thrombocytopenia two weeks after first nivolumab administration. His platelet count initially increased following IV steroids and IVIG treatment. However, it decreased when he was tapered to oral steroids. Pulse IV steroids and one dose of rituximab were given which stabilized his platelet count prior to hospital discharge. Resolution of thrombocytopenia (not shown) was ultimately achieved following two additional doses of rituximab as an outpatient. Shown are the changes in platelet count during his hospitalization. tion of CD8 T-cells, leading to both increased functional activity and frequency. ${ }^{5-8}$ Unrestrained T-cell activation provoked by ICIs can manifest as toxicity in the form of ir-AEs, including ITP as seen in our patient.

In this report, we highlight a case of severe ITP following a single cycle of nivolumab therapy. Previous case reports noted the development of ITP follow2-8 cycles of treatment. ${ }^{5,6}$ ITP is an autoimmune hematologic disorder characterized by isolated thrombocytopenia (platelet count $<100,000 / \mu \mathrm{L}$ ) in the absence of an identifiable cause. The underlying pathophysiology of ITP entails autoantibody production by $\mathrm{B}$ cells resulting in latelet destruction via macrophages in the spleen and liver. T-cell changes include reduction of regulatory $\mathrm{T}$ cell activity and an increase in cytotoxic T-cells. ${ }^{3}$ A majority of patients are asymptomatic, however a minority may present with mild or lifethreatening bleeding. A clinical history is important to rule out other causes of thrombocytopenia as ITP is a diagnosis of exclusion. Examination of the peripheral-blood smear typically shows reduced numbers of platelets. ${ }^{3}$ Increased levels of platelet specific IgG autoantibodies have been reported in previous cases which can provide further support for the diagnosis, but these were not checked in our patient.

Treatment options for ITP include platelet transfusions, which can help to limit bleeding, but its effects are transient. Use of

Conct

glucocorticoids is the standard initial therapy for patients with ITP. Concomitant use of glucocorticoids with IVIG can be associated with a more sustained response. Rituximab, a monoclonal antibody which targets the CD20 antigen expressed on the surface of B-lymphocytes, can be considered in those who do not respond to the above therapies. The main advantage of rituximab is sustained platelet responses that last more than two years in $50 \%$ of patients who have a response. ${ }^{3}$ Our patient experienced complete resolution of ITP following rituximab reaffirming the major role of $\mathrm{B}$ cells in the pathogenesis of ITP.

Of note, our patient had a history of ulcerative colitis prior to nivolumab therapy. While patients with pre-existing autoimmune disease were previously excluded from most ICI clinical trials due to concerns for increased toxicity, a 2019 review by Kennedy et al suggests ICIs may be considered in patients with good control of their underlying autoimmune disorder. This is defined as patients who are either on no immunosuppression or relatively low levels of immunosuppressive therapy. ${ }^{7}$ Unfortunately, despite our patient's ulcerative colitis being well-controlled with no recent flares, he still developed toxicity from immunotherapy.

In conclusion, the diagnosis of nivolumab-induced ITP is challenging given the lack of specific testing and a wide differential diagnosis. In our patient, the clinical course and laboratory results suggested nivolumab-induced ITP given the acute drop in platelets, and recent exposure to immunotherapy. Platelet specific $\mathrm{IgG}$ autoantibodies were not checked but previous reports have noted increased levels which can provide further support for the diagnosis. There are few cases reporting severe ITP following nivolumab treatment. We highlight the importance of recognizing and treating this rare complication of immunotherapy.

\section{References}

1. Bagley S, Kosteva J, Evans T, et al. Immune thrombocytopenia exacerbated by nivolumab in a patient with nonsmall cell lung cancer. Cancer Treat Commun 2016;6:20-3.

2. Calvo R. Hematological side effects of immune checkpoint inhibitors: the example of immune-related thrombocy- 
topenia. Front Pharmacol 2019;10:454.

3. Cooper N, Ghanima W. Immune thrombocytopenia. N Engl J Med 2019;381: 945-55.

4. Delanoy N, Michot JM, Comont T, et al. Haematological immune-related adverse events induced by anti-PD-1 or anti-PD-L1 immunotherapy: a descriptive observational study. Lancet Haematol 2019;6:e48-57.
5. Hasegawa T, Ozaki Y, Inoue T, et al. Nivolumab-related severe thrombocytopenia in a patient with relapsed lung adenocarcinoma: a case report and review of the literature. J Med Case Rep 2019;13:316.

6. Karakas Y, Yuce D, Kilickap S. Immune thrombocytopenia induced by nivolum$\mathrm{ab}$ in a metastatic non-small cell lung cancer patient. Oncol Res Treat
2017;40:621-2.

7. Kennedy L, Bhatia S, Thompson J, et al. Preexisting autoimmune disease: implications for immune checkpoint inhibitor therapy in solid tumors. J Natl Compr Canc Netw 2019;17:750-7.

8. Wei S, Duffy C, Allison J. Fundamental mechanisms of immune checkpoint blockade therapy. Cancer Discov 2018;8:1069-86. 